\title{
CÓMO CONSTRUIR Y SOBREPASAR FRONTERAS ETNOGRÁFICAS. ENTRE ANDES Y AMAZONÍA, POR EJEMPLO
}

\author{
HOW TO BUILD AND OVERTAKE ETHNOGRAPHIC FRONTIERS. \\ BETWEEN THE ANDES AND AMAZON, FOR EXAMPLE
}

\author{
Ricardo Cavalcanti-Schiel ${ }^{1}$
}

\begin{abstract}
Este artículo presenta una discusión sobre los modelos que han configurado el paisaje etnológico de Sudamérica, sus supuestos, criterios y algo de su historia intelectual, sugiriendo que la gran división tierras altas / tierras bajas, lejos de ajustarse a "hechos naturales" responde más bien a miradas analíticas específicas que han movilizado referentes privilegiados (la idea de Estado, por ejemplo) cuyo contenido semántico es casi siempre extrínseco a las lógicas culturales nativas. Sin embargo, si desplazamos el criterio-base de la "organización social" hacia la cosmología, lo que antes había sido visto como distinto por naturaleza puede que encuentre inusitadas continuidades. En este sentido, revisando algunas referencias etnográficas significativas para el caso andino y debatiendo con las proposiciones más recientes de Philippe Descola, tomamos el concepto de "multinaturalismo", acuñado por Eduardo Viveiros de Castro, como un concepto afortunado para expresar esta continuidad de complexiones cosmológicas entre tierras bajas y tierras altas de Sudamérica.
\end{abstract}

Palabras claves: etnología sudamericana, cosmología, Andes, Amazonía, multinaturalismo, animismo.

This paper presents a discussion on the models that have shaped the ethnological landscape of South America, their assumptions, criteria and part of their intellectual history. It suggests that the great divide between highlands and lowlands, far from simply being the translation of "natural facts", responds to specific analytical perspectives that mobilize privileged models (the idea of State, for example) whose semantic content is almost always extrinsic to the native cultural logic. Nevertheless, if we change the basic criterion from "social organization" to "cosmology", what had before been viewed as different by nature may find unusual continuities. In this regard, by reviewing some significant ethnographic references for the Andean case and discussing Philippe Descola's latest proposals, we take the concept of “multinaturalism", coined by Eduardo Viveiros de Castro, as an auspicious notion to express this continuity of cosmological character between the lowlands and highlands of South America.

Key words: South American ethnology, cosmology, Andes, Amazonia, multinaturalism, animism.

El 13 de noviembre de 1939, el suizo Alfred Métraux, residente en Estados Unidos, escribe a su colega norteamericano Julian Steward, que por entonces fungía como investigador asociado en el Bureau of American Ethnology, de la Smithsonian Institution:

Estoy muy preocupado con el hecho de no haber contestado antes, porque el objeto de esta carta es de importancia primordial para mí. He ahí diez años que yo proyecto un Handbook general. Muchas de mis publicaciones me parecen capítulos de esta obra.

En 1932 Nordenskiöld y yo redactamos el plan de un Handbook semejante que yo tenía intención de escribir una vez establecido. Por si acaso el proyecto de usted fuera aplazado de forma indefinida, yo tenía intención de empezar en enero. (...) Estaba decidido a liarme a este trabajo con o sin cooperación. No es una ambición desmesurada: durante estos últimos quince años acumulé muchas notas teniendo en mente a este Handbook (...).

Estoy algo desilusionado por el hecho de que mi parte haya sido reducida a unos cuantos temas (...). ¿Por qué me quitó usted los indios del altiplano a quienes estudié más que cualquier otro de nuestra época? (...) Estoy más que ávido por tratar los mitos sudamericanos e incluso la religión. Son

$\overline{1}$ Departamento de Antropología, Universidade Estadual de Campinas (Unicamp), Campinas, Brasil. riccaval@bol.com.br 
los dos aspectos de las culturas sudamericanas que mejor conozco. Si usted consulta mi bibliografía, verá que tengo cierto derecho a este reclamo (...). El Dr. [Robert] Lowie no se opondrá (...). Yo quiero la mitología más que cualquier otra cosa [énfasis original; traducción nuestra] (Murra 1992:77-78).

Por esos días el mundo se estaba cambiando considerablemente, y (perdonado el cliché) con él, la antropología. No solo la segunda grande guerra había estallado en Europa dos meses antes y empezaba el giro geopolítico que dio origen al mundo contemporáneo que bien conocemos, sino también que el viejo orden colonial que acunara la antropología victoriana entraba en vías de recibir su golpe de misericordia. Como sabemos, antes que esto ocurriera, los británicos tratarían de salvar a las joyas de su corona en el kula científico internacional, otorgando a los linajes centroafricanos el lugar paradigmático de una teoría de la sociedad. Los etnólogos europeos continentales que habían deambulado por tierras del Nuevo Mundo desde fines del siglo anterior, como exploradores asombrados con unos cuantos misterios acerca del "hombre americano", refluirían a sus países de origen o se refugiarían en Estados Unidos. Uno de ellos, Fritz Krause, que emprendiera largos viajes por Brasil central a comienzos del siglo, se alistaría en las filas nazis y vigilaría celosamente a que la obra del judío Franz Boas fuera extirpada de Alemania (Rutsch 2000). Era el fin de la gran tradición etnológica germánica en el ámbito del americanismo. Pero todo esto no quiere decir automáticamente que por fin, en la antropología, América se quedaría para los (norte)americanos. Era necesario algo más que la ausencia (temporal) de los europeos.

En el mismo año de la carta de Métraux a Steward se publicó en Estados Unidos la traducción de la suma teórica de Wilhelm Schmidt para el difusionismo de los "círculos culturales" defendido por la escuela de Viena, con prefacio de Clyde Kluckhohn ${ }^{1}$. Un año antes, sin embargo, el flamante Steward ya dejara patente de forma prácticamente integral su modelo neoevolucionista de la "ecología cultural" con la publicación un estudio de caso (Steward 1938). Las indefiniciones en cuanto a modelos teóricos daban a entrever un campo a conquistar, y por ahí podemos comprender también el trasfondo de la desazón de Métraux.
Asistente del barón Nordenskiöld, exalumno de Paul Rivet, Alfred Métraux -que vendría a ser considerado por Charles Wagley por ocasión de su muerte un cuarto de siglo después como "la más grande autoridad mundial sobre los indios sudamericanos" (Wagley 1964:603)- fuera incorporado por aquél primero (Nordenskiöld) al proyecto que en 1927 Franz Boas y Robert Lowie le encomendaran de un handbook de los indios sudamericanos (Lindberg 1999:xiii), de modo a ampliar hacia Sudamérica la experiencia editorial que Boas y sus alumnos emprendían en Norteamérica ${ }^{2}$. La "encomienda" tenía un valor táctico: congregar a los especialistas europeos que, bajo una genérica y polifacética agenda difusionista ${ }^{3}$, dominaban el escenario de la etnología sudamericana. Sin embargo, el súbito deterioro de la salud de Nordenskiöld seguido de su muerte en 1932, aliado al fracaso de Lowie en obtener financiamiento para el proyecto euroamericano acabaron por inviabilizar el plan inicial. En aquel mismo año de 1932 el National Research Council, por sugerencia de Lowie, había asumido el proyecto del Handbook, pero lo mantuvo intocado hasta el comienzo de la guerra, cuando Julian Steward es encargado de concretar el proyecto, en una confortable posición de manejo de fondos presupuestarios del Comité Internacional para la Cooperación Científica y Cultural, del Departamento de Estado norteamericano (Patterson y Lauria-Perricelli 1999).

Si durante la década de 1920 en los Estados Unidos, los “estudios regionales" (area studies) orientados hacia el exterior habían puesto énfasis en China y Japón, por cuenta de su interés comercial, Latinoamérica va a entrar decididamente en la agenda hacia 1940, cuando es creado un Joint Commitee para estudios sobre la región (Patterson y Lauria-Perricelli 1999:221 $)^{4}$. La publicación del Handbook of South American Indians, bajo la coordinación de Steward (1946-1950) y los auspicios financieros del Departamento de Estado no pueden dejar de ser vistos en este contexto institucional más amplio. Lo que esa obra cargará, por añadidura, es un cambio de agenda intelectual en el que las referencias y preocupaciones europeas son desplazadas o subordinadas a un nuevo marco teórico, que casualmente está a la mano y parece traducir un cierto espíritu científico de la época en Estados Unidos. No se trata de una relación causal simplista, directa y categorial entre geopolítica y teoría antropológica; no se trata de una conspiración; 
sino de una relación mediada por la administración política de prioridades discursivas y temáticas, es decir, por una agenda intelectual ${ }^{5}$.

No obstante, lo que el neoevolucionismo y la "ecología cultural", su primado de la subsistencia y su escala evolutiva del "simple" al "complejo" van a aportar en la aprehensión analítica del paisaje etnográfico sudamericano, es una tipología de áreas culturales estancas, regidas por el criterio naturalizante de la "organización social" como respuesta a la adaptación. El viejo horizonte difusionista, en el que la "cultura material" ofrecía las pistas para suponer la comunicabilidad y el intercambio, dejará de ser sujeto de consideración. Los "complejos culturales" pasan ahora a ser distintos por naturaleza, de modo que el marco neoevolucionista hoy, medio siglo después, puede haber caducado para el particular de los problemas etnológicos de esta o aquella área etnográfica ${ }^{6}$, pero persiste en silencio el mapa de las divisiones que nos legó. Estas divisiones son particularmente evidentes en lo que toca a la separación tierras altas / tierras bajas, sucedánea del marco del Estado como parámetro reificado de la "organización social".

El Estado, en el pensamiento occidental, parece transitar más bien en los avatares de la condición de categoría mítica que en el ámbito "inocuo" de la condición de categoría descriptiva. Por no poder fijar objetivamente el momento -lógico o empírico- en el cual el poder (como generalidad abstracta) empieza a engendrar una institucionalidad de la coerción, el Estado acaba por emerger en el pensamiento social como una inmanencia jusnaturalista. Para los epígonos de la escolástica de Salamanca del siglo XVI, como por ejemplo el jesuita José de Acosta, vivir en "buena policía" significaba conocer (aunque imperfectamente) a Dios y a un rey. Acosta reconoció en Sudamérica a los "reinos" indígenas de la región andina como expresión ejemplar de este conocimiento. Aparte éstos, habían aquellos otros más "bárbaros y silvestres", gente que "vive de su arco y flechas", y que no habría como "reducirlos a policía y obediencia" sino si "sujetados con alguna honesta fuerza" para “enseñarlos primero a ser hombres y después a ser cristianos" (Acosta 1998:453 [1590]). Cuando llega el momento neoevolucionista, el criterio escolástico que había pervivido en el sentido común para operar la distinción entre civilización y barbarie entre los naturales de América se va a proyectar sobre (y como) una presunta escala de la complejización social. Sin embargo, si para el humanismo escolástico (aunque flagrantemente etnocéntrico) las gentes son, por principio, educables (ya que el conocimiento es un atributo humano) -de donde se dedujo el concepto de misión (Huys 1997)-, para la "ecología cultural" las diferencias se inscriben en la naturaleza de los procesos adaptativos, y algo como el Estado se vuelve un fenómeno igualmente naturalizado.

Se hizo clásica en la etnología de las tierras bajas sudamericanas la proposición clastreana de "sociedades en contra del Estado" (Clastres 1974). El bien conocido razonamiento que fundamenta esta proposición es el de que en estas sociedades la reciprocidad conspira permanentemente para impedir la concentración del poder y por consiguiente el establecimiento de instituciones coercitivas duraderas y autorreproducibles. La reciprocidad sería lo que ancla cualquier clase de "poder" a una relación necesaria entre sujetos sociales que preceden su (del poder) especificación, haciendo de su hipotética autonomización institucional una aberración sociológica. De este modo, "sociedades en contra del Estado" no son necesariamente sociedades sin poder-tampoco sociedades sin jerarquía-, pero ellas sólo serían reconocibles porque la reciprocidad, como universal de la regulación, es su antecedente (onto)lógico ${ }^{7}$.

A partir de esta perspectiva, Joanna Overing, confrontando la diversidad de talles morfológicos -es decir, de la organización social- de las sociedades de las tierras bajas, ejemplarmente representada por los tres casos de noroeste amazónico, Guyanas y Brasil central, puesta frente a su "igualitarismo" político común, defiende que, subyacente a esta diversidad, se encontraría el mismo supuesto clastreano de la reciprocidad como precedente ontológico (Overing 1984). Para la autora, en lo que toca a esos tres casos ejemplares, estaríamos frente a "estructuras elementales de la reciprocidad", variaciones -o "transformaciones", como diría Lévi-Strauss en las Mitológicas (Lévi-Strauss 1964)- alrededor de un mismo tema. Sin embargo, sabemos desde los trabajos de John Murra y Franklin Pease, que en los Andes la reciprocidad también es el antecedente (socio)lógico del Estado, y por la misma veta, Juan Ossio le reconocería su condición de precedente ontológico (Ossio 1973) -aunque esta terminología conceptual no esté explicitada en su formulación-. Por cuenta de la división de tareas etnográficas (entre andinistas y amazonistas) entronizada por 
el Handbook, esta clase de reflexión no llegó hasta Clastres. ¿Tendríamos, por consiguiente, que transformar el triángulo de Overing, de las estructuras elementales de la reciprocidad, en un polígono que alcanzara a los Andes? ... ¿como el gran polígono transformacional e indefinido de las Mitológicas, que abarca toda América? O de otra parte ¿deberíamos hablar, para los Andes, de una "estructura compleja de la reciprocidad"? una situación en la que la reciprocidad persistiría como regla, como $a$ priori ontológico, pero donde la complexión social no tiene como límite algún tipo de isonomía. Aquí, al revés, el intercambio reciprocitario produce en un primer momento un cierto tipo de concentración, para en el momento siguiente desdoblarse obligadamente en redistribución ${ }^{8}$, de modo que Franklin Pease (1992:109) pudo sintetizar que "todo el poder es redistributivo en los Andes".

De cualquier modo, nuestro problema no parece estar en el Estado (o en contra de él). Es decir: como "término de verificación"-como quiere la división entre tierras altas y tierras bajas sacralizada por el neoevolucionismo- puede que él sea no más que una ociosidad categorial, justificada tan solamente por nuestras inconscientes compulsiones etnocéntricas.

Aunque contribuyera en el Handbook of South American Indians, Métraux no tuvo en él ninguna clase de protagonismo que llegara a "amenazar" el modelo sufragado por Steward. Sus posibles esfuerzos comparativos se quedaron arrinconados en una sección de cross-cultural studies (el quinto volumen), y destinados a rubros que en este caso resultan particularistas, como religión y chamanismo, ritos, guerra, canibalismo y trofeos humanos; lejos del "núcleo duro" de los criterios más caros a la "ecología cultural"". Asimismo, aunque viniera a aceptar en términos generales el esquematismo tipológico de las "áreas culturales" (Métraux 1982 [1950]) -algo que desde los conceptos difusionistas de "círculos" y "estratos" circulaba en el ambiente etnológico americanista como una especie de lenguaje común-, mantuvo frente a él -o más que todo frente a su pretendida escala evolutiva-considerables reticencias ${ }^{10}$. Haciendo a su manera una suerte de contrapunto al naciente estructural-funcionalismo del otro lado del Atlántico, el Handbook quiso depositar en la "organización social" el criterio por excelencia no solo de reconocimiento del "carácter" de una formación social, sino también de su singularización frente a otras. Sin embargo, ¿y si en su lugar demandáramos, como Métraux, la mitología?...
El término "mitología" sonaría decididamente anticuado si no se reportara a una expresión narrativa formalizada y recurrente. Si de una parte los recursos textuales de la discursividad mítica (sus procedimientos enunciativos, su repertorio de tropos, etc.) constituyen un fenómeno por sí solo digno de atención, por otra parte la clásica distinción entre mythos y logos ya no es para nada pacífica (Latour 1983), de modo que la mitología, más allá de su dimensión textual, es más bien la expresión privilegiada que da paso a lo que hoy día se refiere más comúnmente por cosmología. En la medida en que esta responde como "matriz general de inteligibilidad" del mundo (Viveiros de Castro 1991:179), podemos agregar que ella también nos ofrece un lenguaje privilegiado para la aprehensión de las ontologías específicas, los principios que ordenan el sentido de existencia social acorde a esta $o$ aquella lógica ${ }^{11}$.

Hace pocos años, Philippe Descola propuso un modelo, de alcance mundial, para el reconocimiento de lo que serían cuatro regímenes ontológicos elementales (Descola 2005). La base de su modelo, que es también su hipótesis fuerte, es de que el dualismo de la persona sería un fenómeno universal, es decir, que todas las cosmologías humanas aceptarían que la persona social (no importa si humana o no humana) está constituida por una dimensión física (lo que no excluiría lógicamente su ausencia, como en el caso "ideal" de los "espíritus") -o por así decir, una exterioridad- y una dimensión interior (intrínsecamente inmaterial), responda esta última por cualesquiera de los nombres "intencionalidad, subjetividad, reflexividad, afectos, capacidad de significación o de ensoñación [traducción nuestra]" (Descola 2005:168). Las muchas cosmologías subsidiarían las formas específicas por las que las personas sociales se relacionan en términos de reconocimiento de similitud o diferencia en aquellas dos dimensiones. El modelo sostiene, con la simplicidad necesaria que requieren los modelos abstractos, que las cuatro combinaciones posibles de reconocimiento de similitud y diferencia entre interioridades y exterioridades de las personas sociales definen las "cuatro ontologías", así sintetizadas: (1). el animismo - similitud de las interioridades y disconformidad de exterioridades; (2). el naturalis$m o$ - disconformidad de interioridades y similitud de las exterioridades; (3). el totemismo - similitud de interioridades asociada a similitud de exterioridades; y (4). el analogismo - disconformidad 
de interioridades asociada a disconformidad de exterioridades.

No nos toca aquí discutir la validez o no del modelo, la verificación o no de la hipótesis del dualismo universal de la persona, el rendimiento heurístico de la equivalencia formal entre "identificación” y "relación” (Descola 2005:163-167), o incluso si la operación lógica es la misma cuando se correlacionan las variables en todos los cuatro casos. Lo que nos interesa aquí es la pretensión implícita del modelo en instituir una tipificación de las formaciones sociales análoga a la de las "áreas culturales" o, como indica Descola (2005:289), de que "(...) los esquemas ontológicos se distribuyen por toda la superficie de la tierra según la inclinación de los pueblos hacia esta o aquella forma particular de organizar su práctica en el mundo y con los otros [traducción nuestra]". Para el autor, el régimen ontológico llegaría incluso a establecer posibilidades morfológicas específicas (Descola, 2005:341-385). Así que, insinúa finalmente que el clivaje entre tierras altas y tierras bajas de Sudamérica podría ser recubierto por esta tipificación ontológica en términos de una oposición entre analogismo y animismo, respectivamente.

En el variegado repertorio de referencias etnográficas acopiado en Par-delà nature et culture (Descola 2005), los Andes no llegan a comparecer de forma sistemática. Todavía menos de forma extensiva; lo que vuelve muy temeraria la suposición generalizadora hecha en tres cortos momentos de la obra (Descola 2005:289,317,375), de que esta sería una de las regiones etnográficas del mundo donde "dominan las ontologías analogistas" (Descola 2005:317). Diciéndolo de modo sencillo, esta proposición en realidad carece largamente de base etnográfica. Tomar superficialmente a la descripción de la organización social de los Uru, como está hecha por Wachtel (1990) -o incluso evocar el sistema de ceques del Cusco precolombino-, como recurso indirecto para "constatar" de forma tajante el analogismo como régimen ontológico dominante en los Andes, puede ser no más que una opción por desconocer a todo lo demás de la literatura etnográfica del área, y dejarse llevar por el espejismo de la necesidad de establecer una distinción de naturaleza, ahora sufragada por la ontología, entre las dichas "altas civilizaciones" americanas y los demás indios de a pie. Así que, bajemos a la etnografía.

Los andinos seguramente acreditarían la tesis de la dualidad de la persona. Catherine Allen (1982) registra en Sonqo (Departamento de Cusco) que todas las cosas sobre las que se operan transformaciones son cosas vivas que interaccionan. Los humanos, los cerros, los muertos, las llamas, las papas, son todos sujetos de acción y deseo. Si los cuerpos de todos estos agentes sociales están hechos de materias distintas que pueden ser transformadas, son las voluntades o potencias de unos u otros que operan las transformaciones; transformaciones que pueden ser fundamentalmente de dos clases: las que fertilizan y alimentan, de una parte, y las que enferman, de otra. De este modo, "el mundo material está impregnado, o animado, por una influencia creativa que pasa de objeto a objeto. Todas las cosas materiales, incluyendo a los cuerpos humanos, están conectadas de esa forma y pueden comunicarse una con otra [traducción nuestra]" (Allen 1982:193).

Si más recientemente Alison Spedding (2008:103-126) llegó a sintetizar esta categorización seminal en la imagen de un "paisaje vivificado", ya en 1976 Gabriel Martínez, en su primera etnografía de Isluga (norte de Chile), había sostenido que para la gente aymara de ahí los cerros son entidades vivas precisamente porque dispensan potencia, en una interacción permanente con las demás cosas (Martínez 1989 [1976]:103-105). Las analogías orgánicas del cuerpo y de la vitalidad (ahí incluida la alimentación, la reproducción y el trato social) referidas a esos elementos topográficos también vendrían a ocupar un lugar central en la etnografía de Joseph Bastien (1996 [1978]) sobre los Kallawaya del nordeste del Titicaca, convirtiendo a esos verdaderos personajes etnográficos (los cerros) en referentes paradigmáticos sobre los que se desbordarían las sospechas que vendrían a resultar en la imagen etnográfica de la vivificación generalizada del paisaje a que se refiere Spedding. En efecto, varios de los registros etnográficos que se seguirían a Martínez y a Bastien, subrayarían las capacidades fertilizadoras, curativas y regeneradoras dispensadas por los sujetos topográficos hacia el mundo vegetal, animal y el orden social humano, tanto cuanto la atención que cobran en contraprestaciones rituales. Lo que se conforma en faz a esto es una red de mediaciones ritualmente dispuesta para el trato de las energías dispensadas, que expresa, de otra parte, una suerte de consorcio panagentivo de muchos elementos del ambiente (y de la "sobrenaturaleza"), dotados de ánimo y subjetividad. Esto queda explícito en especial en dos etnografías de los años noventa acerca de la región de Cusco, ya sea para tratar 
de la producción económica (Gose 1994) o de la actividad ritual (Bolin 1998). Por esa misma época, para el ámbito aymara del Altiplano, Hans van den Berg (1989:118-143) sostuvo que la percepción de la "naturaleza" ahí conlleva el reconocimiento de la necesidad de permanentes pactaciones entre los muchos sujetos (es decir, entes dotados de agentividad, voluntad y potencia) que "la compondrían", de los cuales los hombres son tan solo uno de ellos.

Por todo esto, las remisiones al concepto clásico de animismo, depurado ya de su sesgo evolucionista e incluso primariamente proyectivista -el de suponer a los otros sujetos del cosmos como meros calcos de lo humano-, no son ajenas tampoco arbitrarias en la antropología de los Andes (véanse Allen 1982; Haber 2009; Salvia 2011; Sillar 2009). Con todo, una cosa sería remitirse a un fenómeno delimitado, que existiera en pie de igualdad con otros dispositivos epistémicos, como sugirió Núñez del Prado (1970) a propósito del mismo animismo; otra cosa sería remitirse a un régimen ontológico. Lo que nos parece ser el paso del primero al segundo es la propensión del dispositivo a operar en los términos generales de la socialidad y así establecer parámetros universales para las relaciones entre sujetos. En efecto, el cosmos vivificado andino no hace sentido si no está dispuesto como una red de intercambios, regulada por la gramática de la reciprocidad, donde la reproducción de la vida, ya sea la reproducción biológica de los seres o bien la reproducción de las condiciones de subsistencia, depende de los esfuerzos - de su intensidad y su dirección-que unos y otros aplican. El cosmos es resultado de esta conspiración, tanto cuanto de los desencuentros entre sus habitantes; desencuentros que en muchas ocasiones se expresan bajo la forma de una reciprocidad "negativa", la predación, pero que expresa, de todos modos, la precedencia de la condición de sujetos por parte de aquellos que la operan.

Para Descola (2005:280), el analogismo es "el modo de identificación que fracciona el conjunto de los entes existentes en una multiplicidad de esencias, formas y sustancias separadas por tenues intervalos [énfasis nuestra; traducción nuestra]", de modo que todo el trabajo de ponerlos en relación se basa en establecer las similitudes que permitan construir "una densa red de analogías que conexione las propiedades intrínsecas de las entidades diferenciadas [traducción nuestra]". En los Andes, contrastivamente, no se trata de aplicar la verificación de una similitud de atributos dados de antemano, sino de gestionar permanentemente con lo imponderable de las capacidades (e intencionalidades) ajenas. $\mathrm{Si}$ en el analogismo la similitud (ressemblance) es el operador del ordenamiento feliz de la sociabilidad (Descola 2005:285), en los Andes, la diferencia a veces radical de condición, de contingencia física de los sujetos, es lo inmediatamente dado. Entre ellos no hay cualquier razón previa para la existencia de similitudes. Su único atributo común es el animu, la categoría largamente diseminada en el mundo quechua, que parece haber sido un préstamo lexical del castellano. Por ejemplo, como registra Henry Stobart en el norte de Potosí:

La producción agrícola se presenta como una compleja, cambiante e interdependiente red de relaciones, donde el bienestar de los humanos depende de relaciones productivas con una multiplicidad de seres, incluso con los cultivos alimentarios que ellos consumen. (...) Los cultivos alimentarios lloran ${ }^{12}$ del mismo modo que los humanos, como me han explicado, porque ellos tienen апіти: la calidad animada o esencia de todas las cosas vivas, que también es encontrada en ciertos tipos de rocas. La tierra -con sus transformaciones corporales, movimientos hidráulicos y vientos- también es supuesta como llena de animu, así como los cuerpos celestes brillantes, como son el sol, la luna, las estrellas y el relámpago [traducción nuestra; cursivas del original] (Stobart 2006:26-27).

El animu parece más bien una constatación "metafísica" elemental del pensamiento andino -que por ahí se apropió de una voz advenediza para designarla ${ }^{13}$ - que una propiedad distinguible de muchas otras que le serían aproximadas, una propiedad del Ser que se pudiera ponderar o mensurar, y con la que se pudieran establecer analogías diferenciales entre los entes. Si algo en este esquema cosmológico, largamente recurrente en los Andes y fragmentariamente aludido en un sinnúmero de otros registros etnográficos que no los antes mencionados, se acerca a las "cuatro ontologías" de Descola, es el hecho de que aquí los sujetos de la socialidad comparten una misma vitalidad "interior", aunque la complexión física de sus "exterioridades" pueda ser extremadamente diversificada, hasta el punto 
de la no materialidad del alma de los muertos. Es decir, estamos evidentemente más cercanos a un animismo ya reiterado para los Andes -y sugerido en la literatura etnográfica (por Catherine Allen) un decenio antes que Descola empezara a trabajar con este tema en Amazonía- que al esquema del analogismo.

El hecho de que los trabajos de Van den Berg (1989) y Gose (1994) se hayan dedicado al análisis detenido de la dimensión simbólica de las actividades agrícolas, los hizo poner acento en la percepción de que el operador elemental de los intercambios reciprocitarios a la escala del cosmos es el mismo que aquel que se reconoce a la escala de los acuerdos humanos: el dispendio de esfuerzos $-\mathrm{O}$, como ya mencionaba Murra para el campo de la etnohistoria, la entrega de energía (véase la nota 8). En efecto, nuestras investigaciones etnográficas en una región cercana a la de Henry Stobart, la de Tarabuco, en las sierras orientales de los Andes meridionales, nos llevaron a identificar en la categoría kallpa la síntesis conceptual de estas variaciones alrededor de la idea de potencia (Cavalcanti-Schiel 2007, 2014). El campo semántico del término quechua kallpa conjuga las nociones de fuerza, esfuerzo y energía, en el sentido de algo que uno no solamente pueda tener, sino también que hace desprender, que emplea por cuenta de una motivación, y que produce efectos en la economía de las relaciones sociales. Es decir, kallpa no es solo potencia, sino también (o mayormente) la potencia en una acepción dinámica y además ejercida por un agente dotado de ánimo ${ }^{14}$.

En los ritos de trabajo colectivo en Tarabuco -la mink'a y el ayñi-, como en otras partes, el esfuerzo aplicado por uno en la parcela de terreno de otro inscribe en este último una deuda social antes que una deuda personal. Recibir el trabajo de todos implica comprometerse con ellos, entrar a un circuito que delimita una pertenencia social. Entre los Tarabuco, la comunidad inmediata es aquella en donde el intercambio de trabajo entre sus miembros es una expectativa igualmente inmediata. En términos más generales, el ejercicio de esfuerzo es, antes que todo, una partida social abierta que engendra la expectativa de reciprocidad. A la energía aplicada por la Pachamama para hacer crecer los cultivos se le responde ritualmente con una quwa, una incensación votiva. El rito, en sí mismo, es trabajo. Sus elementos materiales, que eventualmente pueden ser subsumidos en el rubro analítico del "sacrificio", son más bien vehículos de la energía que transita, como también, muchas veces, marcadores de una situación de comensalía (Cavalcanti-Schiel 2014). Pero las partidas de la reproducción de la vida jamás se cierran, de modo que hacer una quwa significa entregar a la Pachamama el reconocimiento de estar en deuda y en relación, y esto se hace por medio de un trabajo específico. Sintéticamente, sería plausible sugerir que las obligaciones y transacciones que conforman la sociabilidad en los Andes pueden ser comprendidas como flujos socialmente ordenados de kallpa. Como sabemos, precisamente por cuenta del imponderable de la intencionalidad ajena, estos flujos no siempre son ordenados. El ritual es el lenguaje por excelencia para hacerlo. Incluso la prestación de trabajo humano de unos a otros está enmarcada por prescripciones rituales (la entrega de coca, chicha y cigarrillos, las pausas rituales para descanso, entre otros). Pero aquella fuerza o potencia parece estar siempre en la inminencia de desbordarse. Cuando esto ocurre, sobreviene la calamidad, la enfermedad, el despilfarro, la disgregación, la muerte. Toda la terapéutica en los Andes parece ser el intento de arreglar una energía mal aplicada. En este sentido, es también un negocio de kallpa.

Por supuesto, no es necesario aquí hacer mención a los tantos y ya clásicamente conocidos sujetos de potencia del cosmos andino y su capacidad de entrega de energía, tanto peligrosa cuanto generadora de bienes culturales, materiales $\mathrm{y}$ atributos intelectivos, de unos a otros ${ }^{15}$. Creemos que basta con señalar que su inventario sería ocioso, una vez que potencialmente ampliable al límite de todas las cosas, acorde las circunstancias, o mejor, al reconocimiento "agentivo" de los sujetos (Cavalcanti-Schiel 2007:7). Reconocer a uno como sujeto, o mejor, definir a un sujeto, es reconocerlo como parte de una relación en la que el esfuerzo, fuerza o energía es el significante genérico. Los sujetos no se definen por una suerte de inmanentismo identitario que preceda a su estar-en-relación; por donde nos parece algo desproporcionado, al menos visto desde nuestro escenario etnográfico andino, el planteamiento de Descola (2005:163167) de tomar como lógicamente simultáneas y analíticamente equivalentes la "identificación" y la "relación". La relación nos parece siempre el precedente. Y aquí algunas analogías podrían entonces ser establecidas entre este escenario andino y un otro modelo interpretativo amazónico, ya no el que le toca al animismo de Descola, sino otro 
que le resulta ser algo cercano, pero que en lugar de buscar a invariables de identificación, vuelve a la dicotomía naturaleza/cultura bajo una suerte de radicalidad relacional: el del multinaturalismo de Eduardo Viveiros de Castro.

El concepto de multinaturalismo ha sido fraguado por Viveiros de Castro (2002a) en el contexto de la caracterización que hace de una ontología general amazónica bajo la forma del perspectivismo ${ }^{16}$. Esta idea supone que los sujetos del cosmos -hombres, animales y espíritus- disponen de la capacidad común de ver y percibir los unos a los otros acorde unas cuantas convenciones relacionales recurrentes (caza, alimentación, afinidad, guerra, venganza), pero desde puntos de vista discrepantes, condicionados por las distintas materialidades sustanciales que conforman el habitus particular de cada sujeto:

\begin{abstract}
El perspectivismo no es un relativismo, sino un multinaturalismo. El relativismo cultural, un multiculturalismo, supone una diversidad de representaciones subjetivas y parciales, incidentes sobre una naturaleza externa, una y total, indiferente a la representación; los amerindios proponen el opuesto: una unidad representativa o fenomenológica puramente pronominal, aplicada indiferentemente sobre una diversidad real. Una sola 'cultura', múltiples 'naturalezas'; epistemología constante, ontología variable -el perspectivismo es un multinaturalismo, pues una perspectiva no es una representación.

Una perspectiva no es una representación porque las representaciones son propiedades del espíritu, pero el punto de vista está en el cuerpo [énfasis original; traducción nuestra] (Viveiros de Castro 2002a:379-380).
\end{abstract}

Viveiros de Castro (2002a:357) advierte que el perspectivismo emerge en el contexto de dos características societarias recurrentes en Amazonía, "la valoración simbólica de la caza y la importancia del chamanismo" -aclarando que "se trata de una resonancia simbólica, no de una dependencia ecológica"-, a punto de marbetear el perspectivismo como una "ideología de cazadores" [para todas las citas: traducción nuestra]. Efectivamente, la predación está interesada antes que todo en el cuerpo de la presa (su "espíritu", en este sentido, puede volverse un estorbo), y el objetivo del chamanismo, por excelencia, es abstraerse de una contingencia corpórea para manejar la subjetividad desde otras perspectivas. En un primer momento, eso parece algo distante de los Andes y de la prevalencia, en este segundo caso, de una "ideología de agricultores", centrada en el interés por el esfuerzo ${ }^{17}$, de modo que los acercamientos a las proposiciones del esquema original de Viveiros de Castro no son tan inmediatas, tampoco lineales. Sin embargo, creemos que podemos tomar en serio y en términos literales su planteamiento de que "el perspectivismo es un multinaturalismo". Precisamente porque puede haber otros $^{18}$.

Por evidente, tanto en el perspectivismo amazónico cuanto en el animismo andino, el dispositivo de repartición relacional de los términos naturaleza y cultura es el mismo: al revés del pensamiento occidental moderno, "la cultura o el sujeto serían aquí la forma del universal; la naturaleza o el objeto, la forma del particular [traducción nuestra]" (Viveiros de Castro 2002a:349). Con todo, si en el perspectivismo el cuerpo es el significante genérico de la relación ("el punto de vista está en el cuerpo") en el animismo andino, como decimos antes, este significante es el esfuerzo, fuerza o energía ( $k a l l p a)$.

Otro modo de manejar una confrontación entre estas categorías es hacer uso de un recurso conceptual igualmente caro a Viveiros de Castro, el que respecta al concepto lingüístico de "marca" (Viveiros de Castro 2002b:424-426) ${ }^{19}$. Se puede decir que, en cuanto significantes genéricos, cuerpo y kallpa son valores no marcados. La perspectiva sería el modo marcado del cuerpo, tanto cuanto los atributos de las potencias (la germinación, la fertilidad, el trabajo, la música, los colores o incluso las enfermedades y la muerte) lo son de la energía (kallpa). En ambos casos, "marcar" significa reconocer a un sujeto ${ }^{20}$. La cartografía de estas marcaciones es lo que dibuja el casi siempre ampliable mapa cosmológico -o la cosmografía, por oposición al naturalismo de la geografía- de las muchas naturalezas. El esfuerzo humano es el modo marcado de ese kallpa genérico, por el cual se reconoce a los hombres y mujeres en particular como sujetos de la sociabilidad. De ahí que el trabajo en los Andes (antes que los bienes, las propiedades o incluso el conocimiento enciclopédico) sea el valor ético por excelencia por el que se reconoce la persona (o el agente) social. De modo análogo, la producción social de personas se define igualmente por los esfuerzos realizados sobre su "cuerpo", desde la concepción hasta más allá de 
su muerte -distintamente de Amazonía, donde el intercambio de sustancias conforma la dinámica lógicamente estratégica (Seeger et al. 1978) ${ }^{21}$.

Una consecuencia bastante obvia, por no decir efectivamente un truismo, se desprende de modo común a nuestros dos casos multinaturalistas (el perspectivismo amazónico y el animismo andino). Haciendo su encadenamiento lógico caminar al revés, podríamos decir que si en el pensamiento multiculturalista moderno la alteridad se reporta a la cultura y tiene en este parámetro su término de verificación, en el multinaturalismo, por supuesto, ella se reporta a la naturaleza. Aunque esto suene no más que como un silogismo redundante -y lo es-, que no hace otra cosa que reafirmar las premisas, nos gustaría tomarlo con un operador táctico para enfatizar una vez más las diferencias frente a los avatares del analogismo pretendido por Descola. De ese modo, reiteramos que en el régimen ontológico andino, la cuestión estratégica para los sujetos de la socialidad no es la de administrar la potencialidad de las similitudes (o contigüidad de atributos), sino que tratar permanentemente con la intencionalidad del Otro. Tanto en el perspectivismo amazónico como en el animismo andino, el momento crítico es el del intercambio, es decir: el momento en que la alteridad se pone de frente. Administrar el intercambio de perspectivas en un caso, por medio del trabajo chamánico, corresponde a la regulación del intercambio de energía en otro, por medio del trabajo del ritual. En ambos casos, lo que el negocio estratégico de la socialidad (y de la supervivencia, y de la perpetuación) desvela es una "economía simbólica de la alteridad" (Viveiros de Castro 2002c), y no una pragmática de la identificación.

Esto todo nos lleva a plantear, en síntesis, que el multinaturalismo puede bien ser reconocido como la economía ontológica común a los Andes y Amazonía, sin tener que restringirlo a la forma de una ontología específica de la identificación. No se trata, por consiguiente, de sustituir a la caricatura culturalista de "lo andino", como la dibujó Orin Star (1991) (con sendas repercusiones en el cuadro de la hegemonización reciente de una cierta agenda intelectual en el campo de los estudios andinos), por la de "lo amerindio", sino que restituir a lo andino la dimensión lógico-simbólica a que hace justicia, en un nivel -lo creemos- un poquito más abstracto de lo que usualmente se dispone, pero precisamente, donde ella se encontraría en muy buena posición para dialogar con sus vecinos. Por evidente, esto significa tomar una perspectiva muy distinta de aquella por la que Andes y Amazonía han sido dispuestos como conjuntos socioculturales diferentes "por naturaleza", desde el criterio extrínseco y empiricista de la organización social. Esto significa, al revés, y a ejemplo de Métraux, instar en la "mitología", que no es otra cosa que reconocer en la dimensión cosmológica el cerne intrínseco de la producción de sentido para la propia gente indígena, y de esa forma, desde el desafío "antinarcisista" de las significaciones del Otro (Viveiros de Castro 2010), agregar problematizaciones irremediablemente candentes donde antes parecía regir la placidez (y el silencio) de las verdades al parecer establecidas para siempre.

Agradecimientos: Una primera versión de estas ideas fue presentada en el $54^{\circ}$ Congreso Internacional de Americanistas en la mesa “¿Existe un nuevo americanismo?", coordinada por Dimitri Karadimas y Johannes Neurath, y en las Jornadas de Estudios Andinos 2012, en el Instituto Interdisciplinario de Tilcara de la Universidad de Buenos Aires, en el simposio coordinado por Diego Villar y Pablo Sendón. Agradezco a estos coordinadores la posibilidad de aportar tal discusión en los espacios académicos mencionados. La participación en dichos eventos y la producción del presente artículo ha sido posible gracias al financiamiento de la Fundação de Amparo à Pesquisa do Estado de São Paulo - Fapesp, Brasil, y al generoso diálogo académico establecido con John M. Monteiro, profesor titular de la Universidad de Campinas (Unicamp), fallecido en un trágico accidente cuando la redacción de este texto se acercaba a su final. Agradezco igualmente a los evaluadores de este artículo las apreciadas sugerencias. 


\section{Referencias Citadas}

Acosta, J. 1998 [1590]. Historia Natural y Moral de las Indias. Edición facsimilar. Ediciones de Cultura Hispánica/Agencia Española de Cooperación Internacional, Madrid.

Allen, C. 1982. Body and Soul in Quechua Thought. Journal of Latin American Lore 8(2):179-196.

- - - 1988. The Hold Life Has. Coca and Cultural Identity in an Andean Community. Smithsonian Institution, Washington.

Arnold, D. 2009. Discursos, sujetos e identidades. En iIndígenas u Obreros? La Construcción Política de Identidades en el Altiplano Boliviano, editado por D. Arnold, pp. 47-93. Fundación UNIR, La Paz.

Arnold, D. y J. D. Yapita 2000. El Rincón de las Cabezas. Luchas Textuales, Educación y Tierras en los Andes. Facultad de Humanidades-Universidad Mayor de San Andrés (UMSA)/ Instituto de Lengua y Cultura Aymara (ILCA), La Paz.

Auroi, C. 2004. Fascinación y cansancio: Alfred Métraux en los Andes (1930-1962). Revista Andina 38:253-279.

Bastien, J. W. 1996 [1978]. La Montaña del Cóndor. Metáfora y Ritual en un Ayllu Andino. Traducido por F. Vallvé. Hisbol, La Paz.

Bolin, I. 1998. Rituals of Respect. The Secret of Survival in the High Peruvian Andes. University of Texas Press, Austin.

Canessa, A. 2008. Alteridad y maldad en los Andes meridionales: el caso del kharisiri. En Entidades Maléficas y Conceptos del Mal en las Religiones Latinoamericanas, editado por I. Gareis, pp. 101-118. Institut für Altamerikanistik und EthnologieUniversität Bonn/Shaker Verlag, Bonn/Aachen.

Cañedo R., M. 2013. Cosmopolíticas. Perspectivas Antropológicas. Editorial Trotta, Madrid.

Cavalcanti-Schiel, R. 2007. Las muchas naturalezas en los Andes. Perifèria 7. (23 febrero) http://ddd.uab.cat/pub/ periferia/18858996n7a10.pdf (21 marzo 2013).

- - - 2013. De la Reluctancia Salvaje del Pensamiento. Memoria Social en los Andes Meridionales, en prensa.

Clastres, P. 1974. La Société contre l'État. Recherches d'Anthropologie Politique. Editions de Minuit, Paris.

Descola, P. 2005. Par-delà Nature et Culture. Gallimard, Paris.

Gose, P. 1994. Deathly Waters and Hungry Mountains. Agrarian Ritual and Class Formation in an Andean Town. University of Toronto Press, Toronto.

Haber, A.F. 2009. Animism, relatedness, Life: Post-Western perspectives. Cambridge Archaeological Journal 19:418-430.

Hultkrantz, Å. 1991. Algunas consideraciones acerca del concepto de Nordenskiöld sobre estratificación cultural en América. Boletín del Museo del Oro 30:67-81.

Huys, J.L. 1997. José de Acosta y el Origen de la Idea de Misión. Perú, Siglo XVI. Centro de Estudios Regionales Andinos "Bartolomé de Las Casas", Cusco.

Lara, J. 1971. Diccionario Qhëshwa-Castellano CastellanoQhëshwa. Los Amigos del Libro, La Paz.

Latour, B. 1983. Comment redistribuer le Grand Partage? Revue de Synthèse 110:203-236.
Lévi-Strauss, C. 1964. Le Cru et le Cuit. Plon, Paris.

Lindberg, Ch. 1999 (ed.). Editor's preface. En The Cultural History of the South American Indians, pp. xii-xv. Erland Nordenskiöld. AMS Press, Nueva York.

Lowie, R. 1959. Robert H. Lowie, Ethnologist. A Personal Record. University of California Press, Berkeley/Los Angeles.

Martínez, G. 1989 [1976]. El sistema de los uyuris en Isluga. En Espacio y Pensamiento. I Andes Meridionales, editado por G. Martínez, pp. 13-107. Hisbol, La Paz.

Métraux, A. 1965. Sudamericane Indigene Culture. En Enciclopedia Universale dell'Arte vol. XIII, pp. 343-370. Instituto per la Collaborazione Culturale, Venecia/Roma.

- - - 1982 [1950]. Les Indiens de l'Amérique du Sud. Métailié, Paris.

Murra, J. 1978. La Organización Económica del Estado Inca. Siglo XXI, México D.F.

- . - 1987. ¿Existieron el tributo y los mercados antes de la invasión europea? En La Participación Indígena en los Mercados Surandinos. Estrategias y Reproducción Social. Siglos XVI a $X X$, editado por O. Harris, B. Larson y E. Tandeter, pp. 51-61. Centro de Estudios de la Realidad Económica y Social (CERES), Cochabamba.

- - - 1992. Correspondance entre J. H. Steward et A. Métraux, à propos du Handbook of South American Indians, choisie et traduite par... En Présence d'Alfred Métraux, editado por D. Lecoq, pp. 75-78. Acéphale, Paris.

Nordenskiöld, E. 1912. Une contribution à la connaissance de l'anthropogéographie de l'Amérique. Journal de la Société des Américanistes IX:19-25.

Núñez del Prado B., J.V. 1970. El mundo sobrenatural de los quechuas del Sur del Perú a través de la comunidad de Qotobamba. Allpanchis Phuturinqa 2:57-119.

Ossio A., J.M. 1973. Guaman Poma: Nueva Coronica o Carta al Rey. Un Intento de Aproximación a las Categorías del Pensamiento del Mundo Andino. En Ideología Mesiánica del Mundo Andino, editado por J. Ossio, pp. 155-213. Ignacio Prado Pastor, Lima.

Overing, J. 1984. Elementary structures of reciprocity: a comparative note on Guianese, Central Brazilian, and North-West Amazon socio-political thought. Antropológica 59-62:331-348.

Patterson, T.C. y A. Lauria-Perricelli 1999. Julian Steward and the Construction of Area-Studies Research in the United States. En Julian Steward and the Great Basin. The Making of an Anthropologist, editado por R.O. Clemmer, L.D. Myers y M.E. Rudden, pp. 219-240. The University of Utah Press, Salt Lake City.

Pease, F. 1992. Curacas, Reciprocidad y Riqueza. Fondo Editorial de la Pontificia Universidad Católica del Perú, Lima.

Platt, T. 2002. El feto agresivo. Parto, formación de la persona y mito-historia en los Andes. Estudios Atacameños 22:127-155.

Rutsch Z., M.I. 2000. En torno al Coloquio Mesoamérica y nuestra memoria del doctor Paul Kirchhoff. Dimensión Antropológica 19. http://www.dimensionantropologica.inah.gob.mx/?p=1029 (12 marzo 2013). 
Salvia, D. di 2011. Para una dialéctica de la naturaleza andina. Aproximaciones filosófico-antropológicas a las creencias quechuas en los Apus y la Pachamama. Gazeta de Antropología 27(1), artículo 13. http://www.gazeta-antropologia.es/?p=1334 (20 marzo 2013).

Schmidt, M. 1917. Die Aruaken. Ein Beitrag zum Problem der Kulturverbreitung. Veit \& Comp., Leipzig.

Schmidt, W. 1939 [1937]. The culture historical method of ethnology. Traducido por S.A. Sieber. Fortuny's, Nueva York.

- - - - 1942 [1913]. Ethnologia Sul-Americana. Traducido por S. Buarque de Hollanda. Companhia Editora Nacional, São Paulo.

Seeger, A., R. da Matta y E. Viveiros de Castro 1987 [1978]. A construção da pessoa nas sociedades indígenas brasileiras. En Sociedades Indígenas e Indigenismo no Brasil, editado por J. Pacheco de Oliveira, pp. 11-29. Marco Zero/Editora da Universidade Federal do Rio de Janeiro, Rio de Janeiro.

Sillar, B. 2009. The social agency of things? Animism and materiality in the Andes. Cambridge Archaeological Journal 19:367-377.

Spedding, A. 2008. Religión en los Andes. Extirpación de Idolatrías y Modernidad de la Fe Andina. Instituto Superior Ecuménico Andino de Teología (ISEAT), La Paz.

Starn, O. 1991. Missing the revolution: Anthropologists and the War in Peru. Cultural Anthropology 6:63-91.

Steward, J. 1938. Basin-Plateau Aboriginal Sociopolitical Groups. Bureau of American Ethnology - Smithsonian Institution, Washington (Bulletin 120).

Steward, J. 1946-1950 (ed.). Handbook of South American Indians. Smithsonian Institute, Washington DC.

Stobart, H. 2006. Music and the Poetics of Production in the Bolivian Andes. Ashgate, Aldershot.
Surrallés, A. y P. García Hierro 2004. Tierra Adentro. Territorio Indígena y Percepción del Entorno. IWGIA, Lima.

Uhle, M. 1909. La esfera de influencias del país de los Incas. Revista Histórica IV:5-40.

Van den Berg, H. 1989. La Tierra no da Así no más. Los Ritos Agrícolas en la Religión de los Aymara-Cristianos. Centrum voor Studie en Documentatie van Latijns Amerika (CEDLA), Amsterdam.

Viveiros de Castro, E.B. 1991. Cosmologie. En Dictionnaire de l'Ethnologie et de l'Anthropologie, editado por P. Bonte y M. Izard, pp. 178-180. Presses universitaires de France, Paris.

- - - 1996. Images of nature and society in Amazonian ethnology. Annual Review of Anthropology 25:179-200.

- - - 2002a. Perspectivismo e multinaturalismo na América indígena. En A Inconstância da Alma Selvagem, editado por E. Viveiros de Castro, pp. 345-399. Cosac \& Naify, São Paulo.

- - - 2002b. Atualização e contra-efetuação do virtual: o processo do parentesco. En A Inconstância da Alma Selvagem, editado por E. Viveiros de Castro, pp. 401-455. Cosac \& Naify, São Paulo.

- - - 2002c. O problema da afinidade na Amazônia. En A Inconstância da Alma Selvagem, editado por Eduardo Viveiros de Castro, pp. 87-180. Cosac \& Naify, São Paulo.

- - - 2010. Metafísicas Caníbales. Líneas de Antropología Postestructural. Traducido por S. Mastrangelo. Katz Editores, Buenos Aires/Madrid.

Wachtel, N. 1990. Le Retour des Ancêtres. Les Indiens Urus de Bolivie XXe-XVIe. Siècle. Essai d'Histoire Régressive. Gallimard, Paris.

Wagley, Ch. 1964. Alfred Métraux 1902-1963. American Anthropologist, new series, 66:603-613.

\section{Notas}

1 Se trata de la traducción de Sylvester Sieber del Handbuch der Methode der kulturhistorischen Ethnologie, recién publicado en 1937. Su ambición generalista y su llamamiento a la aprehensión de "multicontinental culture circles" (Schmidt 1939:204 y ss.) lo parece haber hecho poco atractivo para el campo de las preocupaciones estrictamente americanistas. A esto, Schmidt se había dedicado en 1913, en un largo artículo publicado en el Zeitschrift für Ethnologie, de Berlín, que tuvo como única traducción para otra lengua la que le hizo Sérgio Buarque de Hollanda para el portugués, publicada como libro (Schmidt 1942), que no logró impacto siquiera en la muy incipiente etnología brasileña, quedando como una suerte de rareza bibliográfica de anticuarios. En consecuencia de estos desencuentros y de las barreras lingüísticas, la versión de Nordenskiöld (1912) para la teoría difusionista en el continente americano parece haber logrado más receptividad (Hultkrantz 1991).

2 Lowie declinaría en favor de Nordenskiöld y Rivet la idea misma del Handbook de los indios sudamericanos, remontándola a 1924 (Lowie 1959:125).

3 Esto incluía desde las proposiciones de Max Uhle acerca de las influencias que ejerciera y sufriera el "país de los Incas"
Uhle (1909), hasta las permanentes especulaciones sobre los significados de la dispersión arwak (con sus curiosas aproximaciones a aspectos de las sociedades andinas), que encontró en el comparatismo de Max Schmidt su análisis más innovador (Schmidt 1917). Hasta el momento, esta última obra solo se encuentra publicada en alemán. Una traducción (no publicada) al portugués se encuentra disponible en: http://biblio.etnolinguistica.org/schmidt-1917-aruaques.

4 Luego de rematarse el contexto de la Segunda Guerra y ya en el escenario de la guerra fría, van a ser creados Joint Commitees para el Sudeste Asiático y para Estudios Eslavos.

5 Algo que siguen haciendo hoy las agencias norteamericanas en Latinoamérica; la Fundación Ford, por ejemplo.

6 Véase Viveiros de Castro (1996) para un consistente debate sobre el caso de Amazonía.

7 Clastres (1974:175): "Si parece todavía imposible determinar las condiciones de aparecimiento del Estado, se puede en cambio precisar las condiciones de su no aparecimiento [traducción nuestra]".

8 Podríamos llegar a decir que si en las tierras bajas el jefe, por obligación, concentra palabras, en las tierras altas el kuraka, por prerrogativa, concentraba bienes. Sin embargo, 
como nos recuerda Murra (1978:139-149; 1987), la economía de bienes no resultaba ser un campo autonomizado (como quiere, por ejemplo, la economía liberal), sino sucedánea de un término más universal que la precedía: el intercambio de esfuerzos o "energías". Si la figura de los kurakas desapareció históricamente, el fundamento del intercambio de esfuerzos -como vamos a exponer adelante- no.

9 Más informaciones acerca de las "rarezas" alrededor de la marginación de Métraux en el Handbook se encuentran en Auroi (2004:263).

10 Por ejemplo: "El contraste entre la civilización andina y las culturas de los pueblos de la selva o de las pampas argentinas no fue ciertamente tan fuerte como se quiso sostener. Estos últimos, por no haber creado Estados ni fundado ciudades, no vivían en completa barbarie [traducción nuestra]" (Métraux 1965:343).

11 Echamos mano de esa cómoda reconversión terminológica de la "mitología" en "cosmología" no para "anular" al mito, reduciéndolo a mero ingenio discursivo apropiable y manejable por razones "agentivas" ajenas (historicistas incluso), sino, bien al revés, para reconocerlo como expresión de la específica racionalidad fundadora de toda inteligibilidad de la existencia para los que en ella se mueven, a la cual las demás racionalidades operatorias (incluso las eventualmente reconocidas -desde afuera- como "históricas") se subordinan. Y se subordinan por una razón muy sencilla: la inteligibilidad misma de las acciones y relaciones sociales, para sus propios agentes. Suponer a una racionalidad operatoria como universal y precedente (la racionalidad utilitaria del interés, por ejemplo, como les gusta a todos los liberales, antropólogos o no -aunque bajo la escusa de su gramaticalización en un discurso de la "resistencia") sería tan solo una notable manifestación de etnocentrismo colonial, a que Viveiros de Castro (2010:17) muy apropiadamente calificó como "el ambiente sofocante del Mismo".

12 La referencia que hace Stobart a "llorar" (weep) corresponde al verbo quechua waqay, que se reporta primariamente al sonido producido, ni tanto al estado de espíritu, y sirve tanto para el llanto como para el canto de los pájaros cuanto para el sonido producido por los instrumentos musicales.

13 Catherine Allen identificó en su caso etnográfico particular de Sonqo, el uso de la voz quechua sami para expresar la idea de una fuerza vital (Allen 1988:49-54). Sin embargo, las variaciones semánticas alrededor de este término lexical no lo hacen tan genérico y recurrente cuanto animu. Jesús Lara, por ejemplo, desde la perspectiva del quechua meridional, traduce sami como "ventura, fortuna, contento, éxito" (Lara 1971:250)

14 Hasta el momento nos parece que la sola etnografía que trata de esta categoría en términos sistémicos (aunque esté presente en un sinnúmero de vocabularios quechuas de los últimos cuatro siglos ) es la que hicimos en Tarabuco. Debo a Denise Arnold (que formó parte del tribunal de tesis en que esa etnografía fue presentada) la observación de que al término quechua kallpa le correspondería la noción aymara ch'ama (Arnold 2009:85).

15 Aunque la perspectiva de la gente andina sea interesadamente antropocéntrica, resulta plausible para cualquiera que el intercambio de kallpa ocurre entre cualesquiera que sean los sujetos. Esto se vuelve particularmente reconocible en los procedimientos rituales donde entran mediadores.
Esos mediadores pueden ser no solo agentes diversos, sino que también los propios humanos, como es el caso que se constata entre los Tarabuco, en que los humanos hacen la mediación entre los animales domésticos y los "espíritus" en los ritos de marcación de ganado, con fines a granjear fertilidad a los primeros (Cavalcanti-Schiel 2014, cap. 3).

16 La proposición inicial de estos conceptos ha sido hecha en 1996, pero aquí haremos referencia al ensayo en su versión revisada de 2002. Él fue traducido al castellano por Rosa Álvarez y Roger Sansi, y publicado en Surrallés y García Hierro (2004:37-80) y en Cañedo (2013:417-456).

17 En los Andes, los esfuerzos culturales parecen centrados ni tanto en el reconocimiento ubicuo de la predación, sino más bien en su delimitación (sobre todo bajo el marco ritual), eventualmente su domesticación o, en fin, su evitación. Sin embargo, la predación potencial no deja de ser un horizonte latente de la socialidad. Aunque no sea un horizonte inmediato, conforma de modo igualmente genético su campo (Arnold y Yapita 2000; Cavalcanti-Schiel 2014). Así, la "metafísica de la predación" (Viveiros de Castro 2010) en los Andes parece desplazarse de una centralidad pragmática (amazónica) hacia una "subterraneidad" cosmológica, aunque persista tan significante cuanto la primera. En efecto, la predación paradigmática en los Andes es la que se reconoce frente a potencias que guardan alguna forma de indexación al "mundo de abajo" (el ukhu pacha), ámbito por antonomasia de la alteridad, y por ende a su muchas veces débil y dubio régimen de visibilidad y corporeidad (Platt 2002; Canessa 2008). Si se puede efectivamente ilustrar la diferencia entre un caso y otro por las metáforas de una "ideología de cazadores" amazónica y de una "ideología de agricultores" andina -sin que esto signifique una atribución de sustantividades identitarias-, a buen seguro las disposiciones relacionales en cuanto a la "metafísica de la predación" deben ser tomadas en cuenta.

18 Otro modo de decirlo es reiterar la sugerencia de Viveiros de Castro (2010:52) de que "se puede pasar de la noción todavía semiepistemológica de perspectivismo a la noción verdaderamente ontológica de multinaturalismo".

19 El concepto lingüístico de marca lo debemos a los teóricos de la escuela de Praga, Trubetzkoy y Jakobson. Un término no marcado se reporta a un valor genérico, que comporta una significación más amplia, mientras un término marcado es aquel que está especificado y tiene una significación restringida. Vale la observación deportiva de Viveiros de Castro (2002b:424-425): "No se debe olvidar que, en la oposición marcado/no marcado, 'marcado' es, desafortunadamente, el término lexicalmente no marcado, mientras 'no marcado' es el marcado [traducción nuestra]".

20 Para que no queden dudas etnográficas a los andinistas, nombramos aquí a los sujetos paradigmáticos a que clásicamente se ubican esos atributos que nos sirvieron de ejemplo en los paréntesis precedentes: respectivamente, la Pachamama, los ancestros, los hombres, el Tata Sirinu, el Tata K'uychi y, finalmente, para los dos últimos atributos, las entidades liminales entre los pacha (el ukhu y el kay pacha), los condenados, los pichtaqus o cualquier entidad bajo la calificación circunstancial saqra (Cavalcanti-Schiel 2014). Sin embargo, como sabemos, no se trataría siquiera de especificar a un panteón de sujetos exclusivos, porque la ambigüedad es casi siempre 
la norma que mueve las narrativas míticas, tanto cuanto el poder de los chamanes.

21 Para ambos casos, vale la síntesis de Viveiros de Castro (2010:30): "la praxis europea consiste en 'hacer almas' (y en diferenciar culturas) a partir de un fondo corporal-material dado (la naturaleza); la praxis indígena consiste en 'hacer cuerpos' (y en diferenciar las especies) a partir de un conjunto socioespiritual dado: dado precisamente en el mito". 
